\title{
WEB SURVEY DATA AND COMMUTER MODE CHOICE ANALYSIS USING ARTIFICIAL NEURAL NETWORK
}

\author{
Minal Srivastava ${ }^{1}$, Chalumuri Ravi Sekhar ${ }^{2}$ \\ ${ }^{1,2}$ Transportation Planning Division, CSIR-Central Road Research Institute, New Delhi 10025, India
}

Received 26 March 2018; accepted 7 June 2018

\begin{abstract}
Dealing with the present bottlenecks as well as creating long lasting and sustainable transport systems has been the greatest challenge of urban transport planning. Calibrating the present need and forecasting the future demand is the underlying agenda of travel demand forecasting. Mode choice forms an integral part of this process as it gives a complete insight to the mode choice preferences of the commuters validating the introduction of new transport systems to existing ones. This study aims at modelling the mode choice of commuters in National Capital Region of Delhi in India. The data collected for the study was not through the conventional household survey but through a technique of Web-based survey. This survey was hosted at the site of Central Road Research Institute (CRRI) and reached out to people from different walks of life and with varying socio-economic characters. The survey, released in the month of February, 2013 collected about 100 responses and after sifting 94 responses were considered for the analysis. The present study uses the most prominent discrete choice model such as Multinomial Logit (MNL) and a non-conventional machine learning method Artificial Neural Network (ANN) for mode choice analysis. This sample was utilised for developing MNL models using NLOGIT econometric software. The ANN models were configured separately in MATLAB neural network tool box. The results shows that the model developed by ANN is the superior of the two due to higher accuracy and better exploratory power.
\end{abstract}

Key words: mode choice, web survey, artificial neural network, logit model.

\section{Introduction}

Transportation community is bound to face challenges that are both dynamic in nature and futuristic in its application perspective. Amongst the various confluences in Transportation system, congestion is by far the most common and difficult factor to overcome. Congestion drastically affects the level of service of the transport system leading to consequences like delay, accidents which lead to huge economic loss every year. To help alleviate the situation an attempt to model the travel mode choice preferences of commuters in Delhi has been done in this research. The ultimate interest lies in being able to predict the decision making behaviour of the commuters while taking under consideration the attributes of different modes like cost, safety, convenience and travel time. The major modes of transport used in Delhi are private Cars, Two wheelers,

1 Corresponding author: minal.crri@nic.in 
Bus, Metro and Auto. The objective behind mode choice model is to effectively manage the demand and be able to provide for these demands by making changes in the existing system.

Delhi with a population of 22 million (Census of India 2011) is under constant need of expansion of existing transport facilities. Attracting the users of private modes to mass transport modes like Bus and Metro seems to be a solution but is not very feasible given the comfort factor of Mass transport facilities. Therefore relative influence of various parameters associated with different modes has been found out in this study. The mode choice model used in the study is disaggregate model that captures the behavioural aspect of decision maker. Data collection has been tried with an unconventional approach of using Web based survey. This survey was released on the website of CRRI and has been attempted by residents of Delhi and National Capital Region (NCR) with different socioeconomic backdrops, age and gender. The mode choice model was developed based on discrete choice analysis in which models based on Multinomial Logit model (MNL), Probit Model and Nested Logit (NL) were developed. Also soft computing techniques like Artificial Neural Network (ANN) were employed in developing mode choice models. The results of all the above models have been analysed and compared.

\section{Literature Review}

Approach to mode choice modelling varies amongst the transport planners. Mode choice model by (Adams, 1959) is one of the first modal split models to be advised. Earliest research on disaggregate mode choice models was done by (Warner, 1962).
The disaggregate models and its associated advantages over aggregate models, has led to the widespread use of disaggregate discrete choice methods in travel demand modelling, destination choice (Bhat, 1998; Train, 1998), route choice (Gliebe and Koppelman, 2002; Mozolin et al., 2000), air travel choices (Proussaloglou and Koppelman, 1999) activity analysis (Wen and Koppelman, 2000) and auto ownership, brand and model choice (Bhat, 1998). The advantages and useful properties of disaggregate models was presented by other studies (Ortuzar and Willumsen, 2002; Koppelman and Bhat, 2006)

Logit model is one of the best known stochastic choice model (Mc Fadden 1974; Kanafani, 1983; Ben-Akiva and Lerman, 1985). It estimates the proportion of trip makers who choose available mode types based on given conditions or utility criteria. Logit model is often used to compare with other techniques, due to its ability in analysing the trip maker behaviour (Dougherty, 1995; Cantarella and de Luca, 2003; Hensher and Ton, 2002; Nijkamp et al., 1996; Hensher and Ton, 2000). However, the Logit model has certain drawbacks like requirement of large sample size and restriction on dependent variable to be of discrete dataset. The predictive ability of this method also can drop drastically when the number of alternatives increases (Rao et al., 1998). In addition, some researchers claim that this technique is only able to capture the uncertainty in choice decisions and is unable to capture the impact of imprecision in the data (Dell'Orco et al., 2008). In India, most of the researches are oriented towards the use of economic theory of utility maximisation (Chaei, 1976; Minal and Chalumuri, 2016), while few studies have used the concept of disutility minimisation (Rao, 1998). (Parida, 
1994) has developed stated and revealed preferences approaches for modelling homebased work trips in Delhi.

The use of Artificial Intelligence techniques such as Artificial Neural Network (ANN) in Travel demand modelling began in 1960. However it wasn't used for about next three decades in such study due to its weakness, namely the slow response to the modification of inputs despite its extraordinary success at learning or recognising pattern. Neural networks have been used in the transportation demand forecasting by (Chin et al., 1992) and forecasting intercity flows by (Nijkamp et al., 1996). (Rao et al., 1998) has showed Artificial Neural Network advantages in use for traffic behavioural analysis. Forecasting by ANN is done by minimising an error term indicated as the deviation between input and output through the use of specific training algorithm and random learning rate (Black, 1995; Zhang et al., 1998). The theorem proved by (Hornik et al., 1989) and (Cybenko, 1989) states that a multilayered feed forward neural network with one hidden layer can approximate any continuous function up to a desired degree of accuracy provided it contains a sufficient number of nodes in the hidden layer thus they can be considered as universal approximates. (Xie et al., 2003) considered two data mining methods, namely learning tree algorithm and neural networks (back propagation) to improve performance of mode choice forecast. (Golias and Karlaftis, 2001) proposed a recursive partitioning methodology for individual mode choice prediction. The methodology is based on tree-structured nonparametric classification technique. (Edara, 2003) developed mode choice models using artificial neural networks. Data mining procedures like clustering are used to process the extracted data.

\section{Web Survey Data}

The National Capital Region (NCR) which includes New Delhi and the neighbouring cities of, Gurgaon, Faridabad, Ghaziabad, Noida and Greater Noida was chosen as the study area because of the availability of a number of competing modes in the total transport network. Five modes considered in the study were Car ,Two Wheeler, Bus, Metro and Auto. As Delhi has sizeable population and a good prevalence of social media (Facebook) and e-mailing services amongst the residents, an online survey was conducted in the month of February, 2013 and had received till date 98 samples. The survey in the form of a questionnaire were prepared and published in the CRRI website. The questionnaire consisted of four sections namely household Information, personal information, commute Information and mode choice information. Household Information and personal information collected socio-demographic data such as age, gender, household size, household income, number of vehicles in the household, marital status, education status, zone of residence in NCR region of the respondent. Commute Information, and Mode Choice Information were designed to gather information about the mode choice, the purpose of trip, the total cost involved and the time taken in the commute of the respondent. The online survey form was circulated on popular social media sites and e-mailing services targeted at random citizens from Delhi and regions around Delhi. This survey was published after running a "Beta Test" or a "Pilot Survey", that returned acceptable results. A total of 98 responses were received till the month of May out of which 94 samples were chosen after filtering some undesirable responses. 


\section{Mode Choice Model Analysis}

The mode choice modelling has been done through two models namely Multinomial logit model and Artificial Neural Network (ANN) model. The input variables used in the models are In-vehicle Travel Time (IVTT), Out-Vehicle Travel Time (OVTT), Travel Cost (TC) and Household Income (HHINCM). The results from both the models are analysed and compared.

\subsection{Multinomial Logit Analysis}

Logit model is the most commonly used disaggregate model. As a tool it is more transparent and offers a better understanding between the choice decision of commuter and the influence variables. In mode choice analysis, gumbell and normal distribution are the most commonly used probability distribution. These distributions lead to development of the logit and probit model respectively. Though these models are numerically quite similar, logit model is analytically more tractable (Ben-Akiva and Lerman, 1985). The logit model assumes that each individual makes selection from a set of alternatives, often referred to choice set. The functional form of the model for the multinomial Logit Model (MNL), Eq. (1):

$$
P_{o}=\frac{e^{U_{o}}}{e^{U_{o}}+e^{U_{p}}}
$$

Where Po $=$ probability of choosing mode o (own) and Uo and Up are utility functions for own mode and public transport respectively. This utility is normally expressed as a linear function of the characteristics of the individual traveler and attributes of the transport system such as travel cost, travel time etc. In this study, total of five influence variable were considered as discussed in section 3 . The utility functions for own mode (Uo) and Public transport (Up) are given below.

The Logit analysis has been performed by adopting Limdep software which is an econometric software developed by Econometric Software, Inc. The results obtained by MNL model is presented in Table1. The value of coefficient for the different independent variables show the amount and type of influence each of them have on the dependent variable i.e Mode choice. From the results it can be inferred that In-vehicle travel time has a negative influence in case of public mode compared to private modes like Car and Two-wheelers. Travel cost shows no detrimental effect on private mode users as they seem to belong to a higher income group especially the Car users as well as they attach more importance to travel comfort than the cost. Out of vehicle travel time negatively impacts the modal share in each alternative mode except metro and auto mode. This is mainly because of comfort, safety and better service in terms of frequency and accessibility provided by these modes. The goodness of fit of the model can be measured by the parameters depicted in Table 2. The McFadden's R2 value obtained by the model is 0.1325 which is not a very strong fit. McFadden suggested that a value of $\mathrm{R} 2$ between 0.20 and 0.40 gives an excellent fit (McFadden, 1973). The Chi-squared value of model is 32.41 with a degree of freedom of 19 , it indicates that the explanatory power of model has improved over a model where only constant terms are considered. 
Table 1

Multinomial Logit Model Results

\begin{tabular}{|l|l|l|l|}
\hline Name of the Parameter & Coefficient & Standard Error & t-statistic \\
\hline A_CAR & -0.49458 & 1.56881 & 0.315 \\
\hline A_TW & 0.50232 & 1.39287 & 0.3606 \\
\hline A_BUS & 2.10490 & 1.28142 & 1.6426 \\
\hline A_METRO & -1.24435 & 1.44194 & 0.8629 \\
\hline IVTT & -0.03466 & .02560 & 1.3539 \\
\hline OVTT & -0.00967 & .04649 & 0.208 \\
\hline TC & 0.02749 & .02583 & 1.0642 \\
\hline CAR_HHINC & $0.43676 \mathrm{D}-04$ & $.1742 \mathrm{D}-04$ & 2.507 \\
\hline TW_HHINC & $0.15224 \mathrm{D}-04$ & $.1755 \mathrm{D}-04$ & 0.8674 \\
\hline BUS_HHINC & $0.15023 \mathrm{D}-04$ & $.1788 \mathrm{D}-04$ & 0.8402 \\
\hline MET_HHINC & $0.34215 \mathrm{D}-04$ & $.1899 \mathrm{D}-04$ & 1.8017 \\
\hline
\end{tabular}

Table 2

Goodness of Fit for Multinomial Logit Model

\begin{tabular}{|l|l|}
\hline Goodness of fit Parameter & Coefficients \\
\hline Log likelihood function (at convergence) & -106.06147 \\
\hline Log likelihood function (Constants only) & -122.2649 \\
\hline Chi-squared [19 dof] & 32.40694 \\
\hline McFadden's R ${ }^{2}$ & 0.1325 \\
\hline Adjusted McFadden's R ${ }^{2}$ & 0.0760 \\
\hline Number of observations & 94 \\
\hline Akaike Information Criterion AIC & 258.1 \\
\hline
\end{tabular}

\subsection{Nested Logit Analysis}

MNL analysis though widely used in mode choice analysis suffers disadvantage due to its inherent property of Independence from Irrelevant Alternatives (IIA). It's highly flexible nature gives the freedom to include the preferences of commuters and is very useful in modelling repeated choices. It accommodates the similarity between different nests of alternatives in mode choice. The Car and Two-wheelers have been nested under "Private" trunk and Bus, Metro and Auto are nested under
"Public" trunk. The proportion of mode choice for Private mode was obtained as $68.08 \%$ and for Public mode was obtained as $31.92 \%$. Amongst the Private mode Car's ridership was obtained as $52.13 \%$ and $16 \%$ while in Two Wheeler Private mode. Bus obtained the highest ridership of $18 \%$ with Metro and Auto's ridership as $9.5 \%$ and $4.25 \%$ respectively.

The McFadden's R2 and the Adjusted McFadden's R2 values of the model are 0 .1560 and 0.1469 respectively that suggest a reasonably fair fit. 
Table 3

Nested Logit Model Results

\begin{tabular}{|l|l|l|l|}
\hline Name of the Parameter & Coefficient & Standard Error & t-statistic \\
\hline IVTT & 0.03151 & 0.01045 & 3.0153 \\
\hline OVTT & 0.03499 & 0.03333 & 1.0498 \\
\hline TC & 0.00746 & 0.00932 & 0.8004 \\
\hline PRIVATE & 1.70284 & 0.23920 & 7.118 \\
\hline PUBLIC & 1.0 & Fixed Parameter & - \\
\hline
\end{tabular}

\subsection{Artificial Neural Network}

In this study, multilayer feed-forward neural network with single hidden layer was considered along with back propagation algorithm (Dayhoff, 1990; Himanen et al., 1998) for training the network. The ANN analysis for the study was carried out using MATLAB Neural Network Toolbox. A Feed forward multi-layer neural network with Back Propagation algorithm was adopted for classification of mode choice. Out of 94 observation records 66 (70\%) were considered for model training $15 \%$ (14 Sample)were considered for model testing and the rest $15 \%$ (14 sample) was used for model validation. Sigmoid activation function was adopted for both hidden and output layers. The result obtained from the model is presented in Table 4. Optimisation of the network architecture was done to harness the maximum network output with minimum number of neurons in hidden layer. Initially 10 hidden neurons was considered and incremented in step of two hidden neuron till a maximum of 22 hidden neuron. It is observed from the figure that the average minimum MSE decreases by increasing the number of hidden neurons in the hidden layer up to a certain extent.

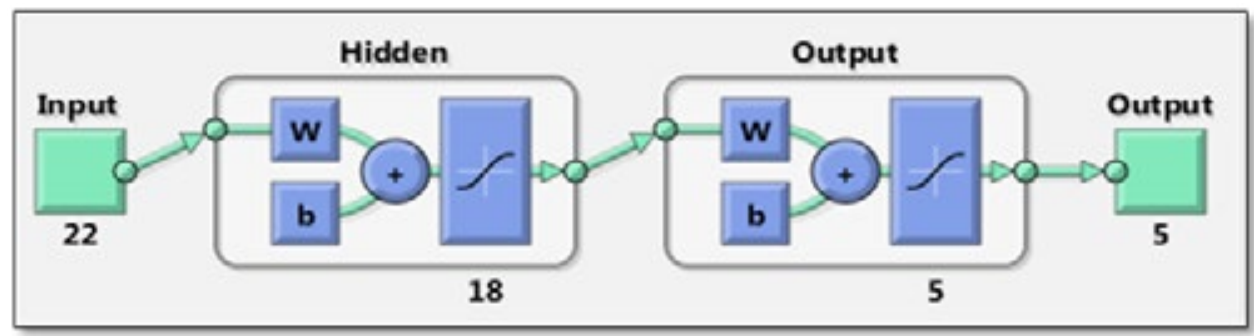

Fig. 1.

Structure of Back Propagation Neural Network (BPNN) Mode Choice Model 


\section{Table 4}

Performance of Back Propagation Neural Network (BPNN) Mode Choice Model

\begin{tabular}{|l|l|l|l|}
\hline Mode Choice ANN Model & Training & Validation & Testing \\
\hline Number of Input Neurons & \multicolumn{2}{|l|}{} \\
\hline Number of Hidden Neurons & 18 & \multicolumn{2}{|l|}{} \\
\hline Number of Output Neurons & 5 & 14 & 14 \\
\hline Sample Size & 66 & $71.4 \%$ & $64.3 \%$ \\
\hline Percentage of Actual Classification & $72.7 \%$ & & \\
\hline
\end{tabular}

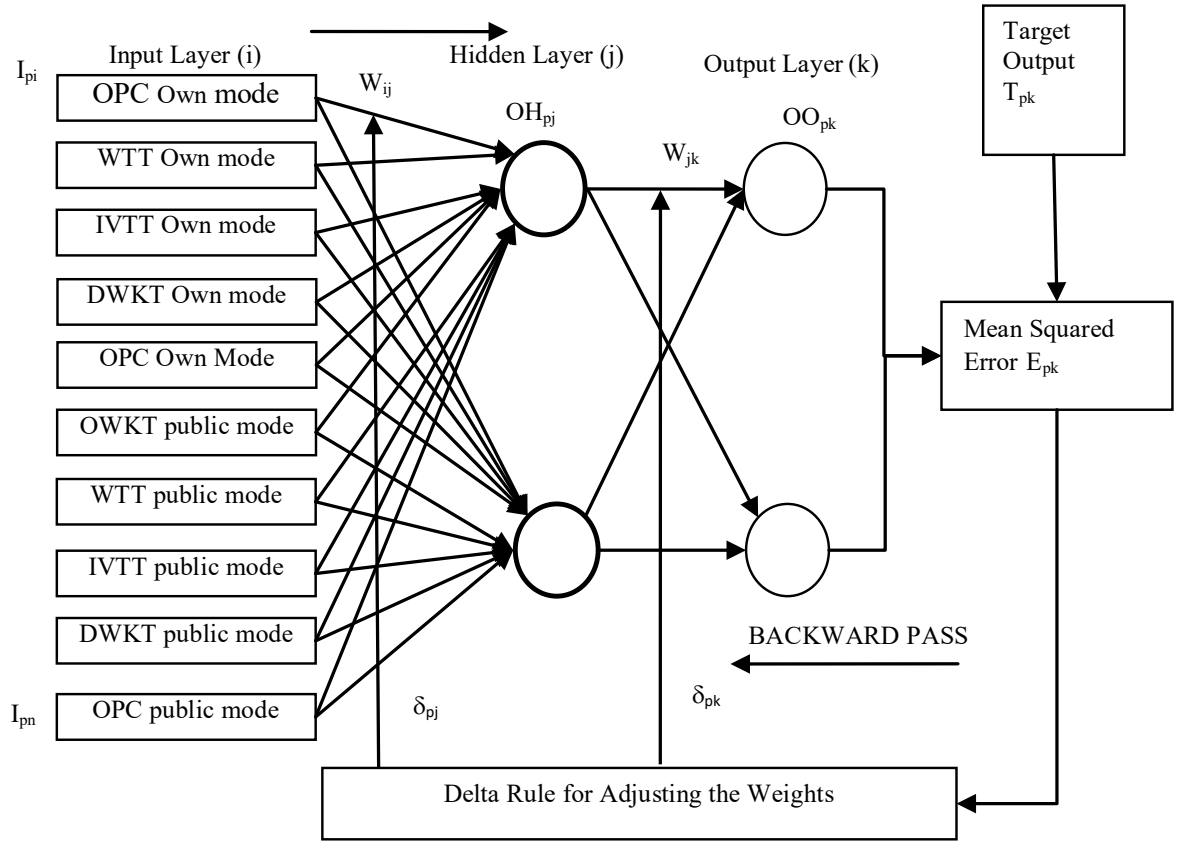

Fig. 2.

Structure of the ANN Model For Modeling Mode Choice Behavior 


\section{Relative Importance of Input Parameters}

Neural Networks are very efficient for solving regression problems with less formal statistical training. It has the ability to implicitly detect complex nonlinear relationships between dependent and independent variables and all possible interactions between predictor variables with the availability of multiple training algorithms. Even though ANN performance is good in predicting the observed output correctly, it suffers from the lack of methods for the interpretation of the significance of input variables. Due to its "black box" nature all the knowledge that the network acquires during learning is implicitly encoded in its numeric connection weights and bias values.

In large networks with multiple layers and large number of neurons and connections, a huge number of numeric values for the weight will result, making extremely difficult to interprets and draw any meaningful explanation for the solution process. In this study, the relative importance of input variables is found by the partitioning of weights algorithm suggested by (Garson, 1991). He presented a technique to interpret the relative importance of each input attribute to the conclusions reached by the network for the purpose of casual analysis. The method essentially involves partitioning the hidden connection weights of each hidden output neuron into components associated with each input neuron. The explanatory variables which were used in model were used to know the relative importance of the input parameters in representing mode choice of commuters. The relative importance of the input parameter in representing mode choice behaviour are presented in Table 4 and the following major observations were drawn from the data.

In vehicle travel time for Two-wheelers and Car users does not affect their ridership which is attributed to the comfort and easy access of these modes. Instead Out of vehicle travel time (mostly comprised of parking time) influences the riders more significantly.

For private mode like Bus, both In-vehicle travel time and Out-vehicle travel time both are significant as accessibility and waiting time for bus users is critical and closely affects its ridership.

For Metro, In-vehicle travel time, Outvehicle travel time as well as cost hold almost equal importance for riders as due to a dedicated right of way it maintains a constant journey speed with high reliability but at a higher cost than buses.

For IPT mode like Auto, the user attaches highest importance to travel time and least importance to the cost. It justifies the objective behind use of IPT i.e. filling up the gap between private and public modes. It provides both spatial and temporal flexibility to the users improving accessibility and reducing waiting time.

Amongst the different socio-economic factors incorporated in the model, Vehicle ownership, Occupation and Household income commuters play important role in mode choice. Higher vehicle ownership and better income group users are more inclined towards the use of private vehicles like car. 


\section{Table 5}

Relative Importance of Explanatory Variables used in ANN Model

\begin{tabular}{|c|c|}
\hline Input Parameters & Importance \\
\hline In-vehicle Travel Time (IVTT) of Car & $3.68 \%$ \\
\hline In-vehicle Travel Time (IVTT) of 2 wheeler & $3.97 \%$ \\
\hline In-vehicle Travel Time (IVTT) of Bus & $5.76 \%$ \\
\hline In-vehicle Travel Time (IVTT) of Metro & $4.14 \%$ \\
\hline In-vehicle Travel Time (IVTT) of Auto & $5.19 \%$ \\
\hline Out -vehicle Travel Time (OVTT) of Car & $5.73 \%$ \\
\hline Out -vehicle Travel Time (OVTT) of 2 wheeler & $4.76 \%$ \\
\hline Out -vehicle Travel Time (OVTT) of Bus & $5.96 \%$ \\
\hline Out -vehicle Travel Time (OVTT) of Metro & $4.50 \%$ \\
\hline Out -vehicle Travel Time (OVTT) of Auto & $5.39 \%$ \\
\hline Travel Cost (TC) of Car & $3.79 \%$ \\
\hline Travel Cost (TC) of 2 wheeler & $3.10 \%$ \\
\hline Travel Cost (TC) of Bus & $3.44 \%$ \\
\hline Travel Cost (TC) of Metro & $4.85 \%$ \\
\hline Travel Cost (TC) of Auto & $2.96 \%$ \\
\hline Age & $4.31 \%$ \\
\hline Gender & $5.09 \%$ \\
\hline Occupation & $5.54 \%$ \\
\hline Household size(Hhsize) & $3.45 \%$ \\
\hline Household Income(Hhincm) & $5.13 \%$ \\
\hline Education & $3.98 \%$ \\
\hline Number of vehicles owned(N_veh) & $5.26 \%$ \\
\hline
\end{tabular}

\section{Comparison of Neural Network and Logit Models}

The performance of ANN frame work is compared to the utility maximisation model based on logit formulation discussed in earlier sections. The former has clearly an advantage compared to Logit models. The comparative performance measured in terms of observations which were correctly predicted by the two approaches is presented in Table 6, Table 7 and Table 8. It is evident from the results that the ANN model, with a classification accuracy of $72 \%$, provides much better results compared to MNL and Nested logit model, with low accuracy of $42 \%$ and $36 \%$ respectively for mode choice amongst Delhi commuters.

It is clearly observed from the results that ANN models are better in modelling behaviour of the commuter in selection of mode compared to the traditional logit model. From the results it can be observed that ANN model has significantly improved performance both in training as well as in validation in comparison to logit approach in all the models except two cases, owning 
cycle group for work trip model and owning two wheeler groups for education model for Visakhapatnam data during validation phase. In this ANN mode prediction accuracy is slightly falls below the logit model predication accuracy. This is mainly due to insufficient data considered during training phase of ANN model. From this it can be conclude that ANN models needs large samples size for modelling the choice behaviour of commuters

\section{Table 6}

Prediction Successive Table For Multinomial Logit Model

\begin{tabular}{|l|l|l|l|l|l|l|l|}
\hline \multirow{2}{*}{$\begin{array}{l}\text { Predicted } \\
\text { Mode Choice }\end{array}$} & Observed Mode Choice & CAR & TW & BUS & METRO & AUTO & TOTAL $\begin{array}{l}\text { Classification } \\
\text { Accuracy }\end{array}$ \\
\cline { 2 - 8 } CAR & 31 & 6 & 6 & 5 & 1 & 49 & $63.26 \%$ \\
\hline TW & 6 & 3 & 3 & 1 & 2 & 15 & $40.0 \%$ \\
\hline BUS & 6 & 3 & 5 & 2 & 1 & 17 & $35.29 \%$ \\
\hline METRO & 5 & 1 & 2 & 1 & 0 & 9 & $55.55 \%$ \\
\hline AUTO & 1 & 2 & 1 & 0 & 0 & 4 & $0 \%$ \\
\hline TOTAL & 49 & 15 & 17 & 9 & 4 & 94 & $42.55 \%$ \\
\hline
\end{tabular}

Table 7

Prediction Successive Table for Nested Logit Model

\begin{tabular}{|c|c|c|c|c|c|c|c|}
\hline \multirow{2}{*}{$\begin{array}{l}\text { Predicted } \\
\text { Mode Choice }\end{array}$} & \multicolumn{7}{|c|}{ Observed Mode Choice } \\
\hline & CAR & TW & BUS & METRO & AUTO & TOTAL & $\begin{array}{l}\text { Classification } \\
\text { Accuracy }\end{array}$ \\
\hline CAR & 27 & 8 & 7 & 5 & 2 & 49 & $55.1 \%$ \\
\hline TW & 8 & 3 & 2 & 2 & 1 & 15 & $20.0 \%$ \\
\hline BUS & 8 & 3 & 3 & 2 & 1 & 17 & $17.64 \%$ \\
\hline METRO & 5 & 2 & 1 & 1 & 0 & 9 & $11.12 \%$ \\
\hline AUTO & 1 & 1 & 1 & 0 & 0 & 4 & $0 \%$ \\
\hline TOTAL & 49 & 17 & 14 & 10 & 4 & 94 & $36.17 \%$ \\
\hline
\end{tabular}

\section{Table 8}

Prediction Successive Table for ANN Model

\begin{tabular}{|l|l|l|l|l|l|l|l|}
\hline \multirow{2}{*}{$\begin{array}{l}\text { Predicted } \\
\text { Mode Choice }\end{array}$} & Observed Mode Choice & TW & BUS & METRO & AUTO & TOTAL & $\begin{array}{l}\text { Classification } \\
\text { Accuracy }\end{array}$ \\
\cline { 2 - 8 } & 48 & 8 & 3 & 6 & 1 & 66 & $72.7 \%$ \\
\hline CAR & 1 & 4 & 0 & 0 & 0 & 5 & $80.0 \%$ \\
\hline TW & 0 & 3 & 14 & 3 & 2 & 22 & $63.6 \%$ \\
\hline BUS & 0 & 0 & 0 & 0 & 0 & 0 & $0 \%$ \\
\hline METRO & 0 & 0 & 0 & 0 & 1 & 1 & $100 \%$ \\
\hline AUTO & 49 & 15 & 17 & 9 & 4 & 94 & $71.27 \%$ \\
\hline TOTAL & & & & & & & \\
\hline
\end{tabular}




\section{Conclusions}

A good data set paves way for better choice models. The conventional method of data collection like Personal interview engages huge resource and time. A novel technique of data collection through a web-based survey has been tried in this study. The feasibility and accuracy provided by such unconventional data set has been tested. The choice model developed for mode choice preference of commuters of National Capital Region (NCR) of India has been attempted and its accuracy has been tested. Multinomial Logit and Nested Logit models were developed to assess the mode choice. Since the classification accuracy of logit models was too low to be considered appropriate, it was also decided to test the applicability of advanced data mining techniques such as Artificial Neural Networks to model mode choice.

In the present study, it can be observed that the developed model utilising online data also demonstrate the similar type of capabilities in terms of prediction of mode choice; therefore it is recommended that the web-based online survey is effectively managing the resources and time which is otherwise hugely consumed in conventional data collection.

A comparative evaluation between Multinomial logit (MNL), Nested Logit and multi layer perceptron artificial neural network models for predicting the choice behaviour for commuters in Delhi based on vehicle ownership shows that the performance of $\mathrm{ANN}$ is much superior to MNL both in calibration and testing.

Through ANN a better understanding of relative importance of different modal attributes and socio-economic attributes can be had.

The Nested Logit model had the lowest prediction accuracy (36\%). It can be attributed to the less number of samples used in the study. Use of larger number of samples may increase the efficiency of Logit models.

It can be safely concluded that $\mathrm{ANN}$ is very useful for exploratory analysis and preliminary examination of possible input variables for other types of models although the "black-box" nature of ANN proves to be a drawback.

Despite of a robust predictive ability of ANN over MNL model, practitioners are more inclined towards using highly transparent statistical methods.

\section{Acknowledgements}

We are very grateful to the Director, CSIRCentral Road Research Institute for allowing us to publish this paper.

\section{References}

Adams, W.T. 1959. Factors Influencing Mass Transit and Automobile Travel in Urban Areas, Public Roads 30(11): 256-60.

Ben-Akiva, M.E.; Lerman, S.R. 1985. Discrete choice analysis: theory and application to travel demand (Vol. 9). MIT press.

Bhat, C.R. 1998. Analysis of travel mode and departure time choice for urban shopping trips, Transportation Research Part B: Methodological 32(6): 361-371.

Black, W.R. 1995. Spatial interaction modeling using artificial neural networks, Journal of Transport Geography 3(3): 159-166. 
Cantarella, G.E.; de Luca, S. 2003. Modeling transportation mode choice through artificial neural networks. In Proceedings of the Fourth Uncertainty Modeling and Analysis, 2003. ISUMA 2003, 84-90.

Chaei, S.R. 1976. Disaggregated transportation demand model for home based trip generations, Doctoral dissertation, Department of Civil engineering, University of Roorkee.

Chin, S.M.; Hwang, H.L.; Miaou, S.P. 1992. Transportation demand forecasting with a computersimulated neural network model. In Proceedings of the International Conference on Artificial Intelligence Applications in Transportation Engineering, 349-390.

Cybenko, G. 1989. Approximation by superpositions of a sigmoidal function, Mathematics of control, signals and systems 2(4): 303-314.

Dayhoff, J.E. 1990. Neural Network Architectures: An Introduction. New York. Van Nostrand Reinhold.

Dell'Orco, M.; Circella, G.; Sassanelli, D. 2008. A hybrid approach to combine fuzziness and randomness in travel choice prediction, European Journal of Operational Research 185(2): 648-658.

Dougherty, M. 1995. A review of neural networks applied to transport, Transportation Research Part C: Emerging Technologies 3(4): 247-260.

Edara, P.K. 2003. Mode choice modeling using artificial neural networks, Doctoral dissertation, Virginia Polytechnic Institute and State University.

Garson, G.D. 1991. Interpreting neural-network connection weights, Artificial Intelligence Expert 6(4): 46-51.

Golias, I.; Karlaftis, M.G. 2001. An international comparative study of self-reported driver behavior, Transportation Research Part F: Traffic Psychology and Behaviour 4(4): 243-256.
Hensher, D.A.; Ton, T. 2002. TRESIS: A transportation, land use and environmental strategy impact simulator for urban areas, Transportation 29(4): 439-457.

Hensher, D.A.; Ton, T.T. 2000. A comparison of the predictive potential of artificial neural networks and nested logit models for commuter mode choice, Transportation Research Part E: Logistics and Transportation Review 36(3): 155-172.

Himanen, V.; Nijkamp, P.; Reggiani, A. 1998. Neural networks in transport applications. Ashgate Publishing Company.

Hornik, K.; Stinchcombe, M.; White, H. 1989. Multilayer feedforward networks are universal approximators, Neural networks 2(5): 359-366.

Kanafani, A. 1983. Transportation Demand Analysis. Mcgraw Hill. 320 p.

Koppelman, F.S.; Bhat, C. 2006. A self instructing course in mode choice modeling: multinomial and nested logit models. Available from internet: <http://www.caee. utexas.edu>.

McFadden, D. 1973. Conditional Logit analysis of qualitative choice behavior, Frontiers in Econometrics 105-142.

Minal, S.; Chalumuri, R.S. 2016. Commuter's sensitivity in mode choice: An empirical study of New Delhi, Journal of transport geography 57: 207-217.

Mozolin, M.; Thill, J.C.; Usery, E.L. 2000. Trip distribution forecasting with multilayer perceptron neural networks: A critical evaluation, Transportation Research Part B: Methodological 34(1): 53-73.

Nijkamp, P.; Reggiani, A.; Tritapepe, T. 1996. Modelling inter-urban transport flows in Italy: A comparison between neural network analysis and logit analysis, Transportation Research Part C: Emerging Technologies 4(6): 323-338. 
Ortuzar, J. D.; Willumsen, L.G. 1994. Modelling transport, 2 edn. John Wiley \& Sons Ltd. West Sussex. England.

Parida, M. 1994. Mode Choice Analysis Based on Stated and Revealed Preferences for Home based work trips in Delhi, Doctoral dissertation, Department of Civil engineering, University of Roorkee.

Proussaloglou, K.; Koppelman, F.S. 1999. The choice of air carrier, flight, and fare class, Journal of Air Transport Management 5(4): 193-201.

Rao, P.S.; Sikdar, P.K.; Rao, K.K.; Dhingra, S.L. 1998. Another insight into artificial neural networks through behavioural analysis of access mode choice, Computers, Environment and Urban Systems 22(5): 485-496.

Rao, S.B.S. 1998. Community Response to Components of Urban Transport for work Journey through Mode Choice Models, Doctoral dissertation, Department of Civil Engineering, NIT Warangal, Warangal, India.
Warner, S.L. 1962. Stochastic Choice of Mode in Urban Travel: a study in a binary choice, North Western University Press, Evanston, Illinoise.

Wen, C.H.; Koppelman, F.S. 2000. A conceptual and methodological framework for the generation of activitytravel patterns, Transportation 27(1): 5-23.

Xie, C.; Lu, J.; Parkany, E. 2003. Work travel mode choice modeling with data mining: decision trees and neural networks, Transportation Research Record: Journal of the Transportation Research Board 1854: 50-61.

Zhang, G.; Patuwo, B.E.; Hu, M.Y. 1998. Forecasting with artificial neural networks: The state of the art, International Journal of Forecasting 14(1): 35-62. 